\title{
SIFT-based Camera Localization using Reference Objects for Application in Multi-Camera Environments and Robotics
}

\author{
Hanno Jaspers ${ }^{1}$, Boris Schauerte ${ }^{2}$ and Gernot A. Fink ${ }^{1}$ \\ ${ }^{1}$ Department of Computer Science, TU Dortmund University, 44221 Dortmund, Germany \\ ${ }^{2}$ Institute of Anthropomatics, Karlsruhe Institute of Technology, 76131 Karlsruhe, Germany \\ \{hanno.jaspers, gernot.fink\}@udo.edu,boris.schauerte@kit.edu
}

Keywords: Camera pose estimation, relative pose, camera calibration; Scale Ambiguity, reference object; Local features, SIFT; multi-camera environment, smart room, robot localization.

\begin{abstract}
In this contribution, we present a unified approach to improve the localization and the perception of a robot in a new environment by using already installed cameras. Using our approach we are able to localize arbitrary cameras in multi-camera environments while automatically extending the camera network in an online, unattended, real-time way. This way, all cameras can be used to improve the perception of the scene, and additional cameras can be added in real-time, e.g., to remove blind spots. To this end, we use the Scale-invariant feature transform (SIFT) and at least one arbitrary known-size reference object to enable camera localization. Then we apply non-linear optimization of the relative pose estimate and we use it to iteratively calibrate the camera network as well as to localize arbitrary cameras, e.g. of mobile phones or robots, inside a multi-camera environment. We performed an evaluation on synthetic as well as real data to demonstrate the applicability of the proposed approach.
\end{abstract}

\section{INTRODUCTION}

In recent years smart rooms have attracted an increasing interest, e.g., to improve the productivity in office environments and assist the personnel in crisis response centers. For this purpose, the identities of the persons in the room have to be determined (see (Salah et al., 2008)) as well as the audio-visual focus of attention has to be estimated (see (Voit and Stiefelhagen, 2010, Schauerte et al., 2009)), e.g. to present personalized information on the display a person is currently looking at. However, these applications rely on the fusion of information that is provided by a set of sensors, most importantly microphones and camera arrays. In order to fuse the information from different sensors in the environment it is necessary to determine their extrinsic parameters in a common coordinate frame. In the following, we focus on cameras as sensors and in this domain offline calibration methods are applied most commonly (see, e.g., (Brückner and Denzler, 2010; Aslan et al., 2008; Xiong and Quek, 2005; Rodehorst et al., 2008)). Unfortunately, these methods usually require a time-consuming manual procedure and need to be repeated if a new camera is added or a camera is relocated.

In this contribution, we analyze how we can use the views of the already calibrated cameras in an environment to localize a new camera and thus enable subsequent sensor fusion. This, for example, can be used to enable easily extensible camera networks, allow the seamless integration of the sensor information of mobile robotic agents, and allow mobile robotic agents to use the information of the sensors that are installed in the environment to enhance their perception capabilities. With the proposed approach, we are able to determine the absolute pose of a new camera in the global coordinate system given only one known camera and an arbitrary reference object. Although our focus on a single known camera may limit the achievable results in scenarios with a huge amount of cameras with widely overlapping views, we chose this focus, because it enables us to integrate cameras that only view parts of the scene that are recorded by only one other camera. This is especially important, if the viewpoints of the cameras are very different or only few cameras are used, which - according to our experience - seems to be more realistic in most application areas. However, our method can naturally be extended to situations with multiple views. To this end, we calculate the poses for all plausible camera pairs and subsequently aggregate the pairwise localization results. In contrast to most previous work, we do not rely on special calibration patterns or devices and use arbitrary reference objects instead ${ }^{1}$ To this

\footnotetext{
${ }^{1}$ However, we could - of course - use existing calibra-
} 
end, only a very small amount of user interaction is required to build an appropriate database of known objects. The necessary information about the reference objects might be automatically collected from the internet, or in the domain of cognitive robots, directly obtained by actively exploring potential objects. Once the information is available, cameras can be localized completely automatic at any time.

\section{RELATED WORK}

The research area of camera calibration, of which camera pose estimation is an important aspect, is a well known and researched topic and accordingly many different approaches have been proposed (see, e.g., (Brückner and Denzler, 2010; Aslan et al., 2008, Frank-Bolton et al., 2008; Xiong and Quek, 2005. Rodehorst et al., 2008)).

In order to calculate the relative pose, the fundamental matrix has to be computed. The normalized and the standard 8-point algorithm, variants of the 7-point algorithm (Hartley and Zisserman, 2004), as well as a 6-point and 5-point algorithm were compared (Stewénius et al., 2006, Nistér, 2004). The normalized 8-point algorithm performed considerably better than the non-normalized version and its use was recommended when no prior knowledge about the camera motion, i.e. sideways or forward motion, is available. The 5-point algorithm achieved better results in most cases, as confirmed in (Rodehorst et al., 2008), but it had problems with forward motion, where the results were worse. We used the 8-point algorithm in our approach because of its overall good results.

For finding point correspondences, we rely on the matching of SIFT features, proposed by Lowe in (Lowe, 1999; Lowe, 2004). In the context of relative pose estimation and scene reconstruction, SIFT has been used before in (Liu and Hubbold, 2006; Snavely et al., 2008).

In previous work, (Xiong and Quek, 2005) described a system to calibrate the intrinsic and extrinsic parameters of camera networks in meeting rooms. They used a box with dots and other markers to calibrate the cameras. This resulted in a good accuracy of less than $1 \mathrm{~cm}$ for the camera positions of most cameras. However, a lot of user interaction is required to perform the calibration: Camera pairs were chosen manually and the calibration box had to be placed for each camera pair specifically.

(Svoboda et al., 2005) proposed a technique with less user interaction for the calibration of a multi-

$\overline{\text { tion patterns and }}$ objects as reference objects as well. camera environment. Instead of using dedicated calibration objects or markers, they used a bright spot as the calibration feature, generated by a laser pointer with a small diffusing piece of plastic attached to it. Their algorithm can be used to fully calibrate the camera network, the only user interaction is waving the laser pointer through the working volume.

Aslan et al. pursued a similar approach to automatically calibrate the extrinsic parameters of multiple cameras (Aslan et al., 2008). Instead of a bright spot, they detected people walking through the room and used a point on top of every person's head as calibration feature. The relative pose is estimated for every camera pair, and with this, the complete camera network is built up using a global error minimization technique. The precision has been evaluated in different indoor scenarios, arriving at a projection error of less than 6px and a triangulation error of markers in the scene of about $5 \mathrm{~cm}$. The positions of the camera centers have not been compared to their ground truth.

Recently, Brückner and Denzler proposed an active calibration technique for multi-camera systems (Brückner and Denzler, 2010). They use the rotating and zooming capabilities of pan-tilt-zoom (PTZ) cameras to optimize the relative poses between each camera pair. The scaling factors in camera triangles are estimated with two of the three relative poses. In contrast to our approach no reference object is required, but the types of cameras that can be used are limited to PTZ cameras. Our system does not put any limitations on the types of cameras, allowing, for example, a combination of fixed PTZ cameras, cameras mounted on robotic platforms and even smartphone cameras. Furthermore, more than two cameras are needed, whereas our approach allows to estimate the absolute pose of only two cameras.

Similar techniques as those used for the calibration of multi-camera environments can be applied to other applications, such as robot indoor localization. In (Frank-Bolton et al., 2008), a system to localize and track a robot based on a set of known views was proposed. First, a set of views of the scene, an indoor environment, was recorded with the robot for specific positions and different orientations. The positions were chosen on a grid, roughly $90 \mathrm{~cm}$ apart. The environment was surrounded by project posters to facilitate the search for image correspondences. FrankBolton et al. come to the conclusion, that epipolar geometry in conjunction with the normalized 8-point algorithm is too sensitive to ensure a robust and accurate pose estimation. Instead, they use a technique called quality threshold clustering, which resulted in an average position error of $46 \mathrm{~cm}$ and a mean orientation error of $9^{\circ}$. Our results (see section 4 ) lead to the 
assumption, that a more precise localization can be achieved with our approach, using the project posters as reference objects.

\section{POSE ESTIMATION}

Our sytem that computes the global pose of a camera in relation to a known camera consists of three main steps (see Fig. 1). In the first step, the relative pose of the camera is calculated. This requires the detection of point correspondences between the two considered images. To this end, SIFT features (Lowe, 2004) are computed and matched. This step may introduce outliers, i.e. correspondences of image points that are not projections of the same scene point. If they are not robustly eliminated, errors in the estimated pose will occur. In the second step we optimize the estimated relative pose in order to minimize the influence of noise and achieve better results. Finally, the global scaling of the relative pose is calculated in the third step. This step is based on the detection of at least one reference object of known size within the scene.

\subsection{Relative Pose}

The relative pose of the second camera is calculated using epipolar geometry. For this, the fundamental matrix $\mathbf{F}$ is calculated with the normalized 8-point algorithm in conjunction with RANSAC, to eliminate outliers from the point correspondences (Hartley and Zisserman, 2004). Using the fundamental matrix the scene can be reconstructed up to a projective ambiguity. We assume calibrated cameras with the intrinsic calibration matrices $\mathbf{K}_{\mathbf{1}}$ and $\mathbf{K}_{\mathbf{2}}$. Accordingly, the reconstruction can be performed up to a scale ambiguity, as illustrated in Fig. 2. As a result of the unknown scale, the translation vector $\mathbf{t}$ is normalized to $\|\mathbf{t}\|=1$.

The essential matrix $\mathbf{E}$, a special case of the fundamental matrix for normalized image coordinates, is obtained as

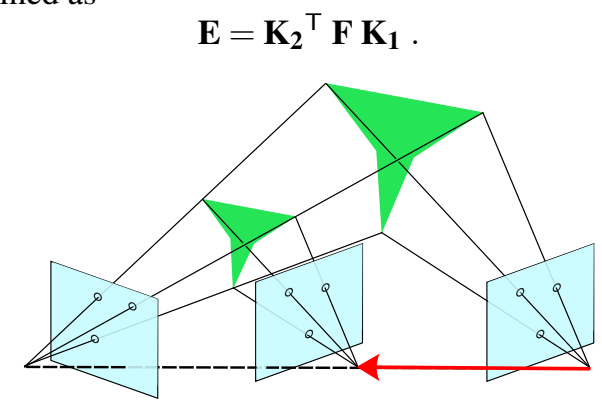

Figure 2: Visualization of the scale ambiguity. The second camera can "slide" along the baseline between the cameras (i.e. different scaling of the relative position), without affecting the point correspondences.

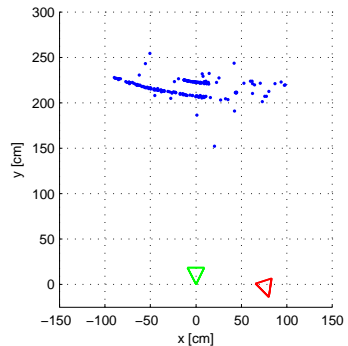

(a)

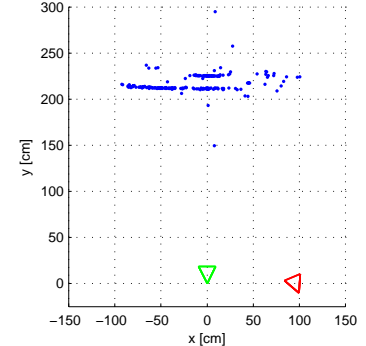

(b)
Figure 3: The relative pose of the red camera is estimated (a) using only epipolar geometry, and (b) with additional nonlinear optimization. With optimization, most of the reconstructed points (blue) are parallel to the $\mathrm{x}$-axis, which complies with the ground truth.

The defining property of the essential matrix is that two of its singular values are equal and the third one zero. Due to the presence of noise that is introduced through small errors in the camera calibration process and the estimation of the fundamental matrix, this property has to be enforced. Thus, let

$$
\mathbf{E}=\mathbf{U} \operatorname{diag}\left(\sigma_{1}, \sigma_{2}, \sigma_{3}\right) \mathbf{V}^{\top}, \text { with } \sigma_{1} \geq \sigma_{2} \geq \sigma_{3}
$$

be the SVD of $\mathbf{E}$. The essential matrix $\widetilde{\mathbf{E}}$, which minimizes the Frobenius norm $\|\mathbf{E}-\widetilde{\mathbf{E}}\|$, is calculated as

$$
\widetilde{\mathbf{E}}=\mathbf{U} \operatorname{diag}(\sigma, \sigma, 0) \mathbf{V}^{\top}, \text { with } \sigma=\frac{\sigma_{1}+\sigma_{2}}{2} .
$$

The essential matrix can be decomposed into four possible solutions for the pose $(\mathbf{t}, \mathbf{R})$ of the second camera, with translation $\mathbf{t}$ and rotation $\mathbf{R}$. There's only one solution for which the reconstructed 3Dpoints are in front of the image planes of both cameras. This constraint is termed cheirality constraint. In ideal circumstances it would suffice to reconstruct one point from a point correspondence pair and to test whether it satisfies the cheirality constraint. But since outliers can't be ruled out a voting mechanism has to be put in place to determine the correct solution: Each reconstructed point "votes" for the solution, that satisfies its cheirality constraint. The solution with the highest number of votes is chosen as the correct solution.

\subsection{Pose Optimization}

Figure 3(b) shows the reconstruction of a scene that was obtained using the relative pose as described above. The camera was oriented towards a flat wall. However, the reconstructed points lay on a curved surface. This indicates small errors in the obtained pose. To improve the relative pose, a nonlinear, Trust-Region-Reflective optimization step (Coleman and $\mathrm{Li}, 1996$ ) has been introduced that minimizes 


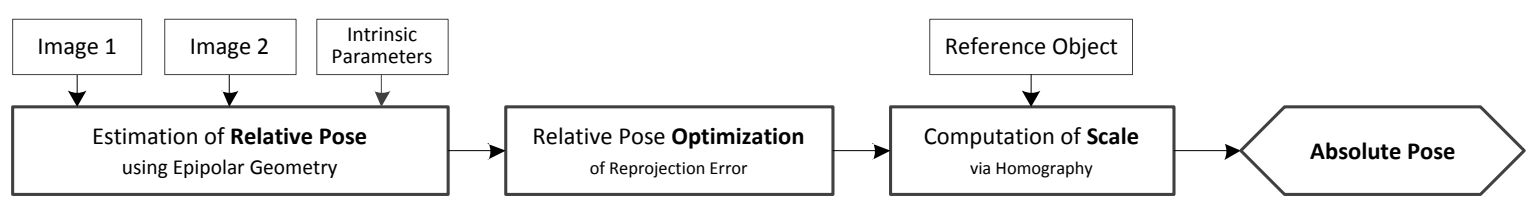

Figure 1: This overview of our approach shows the three main steps to calculate the absolute pose of a camera. At first the relative pose is estimated using epipolar geometry. Afterwards, the relative pose is optimized and then scaled with a scaling factor retrieved from a known reference object, giving the absolute pose of the second camera in relation to the first camera.

the reprojection error. In contrast to LevenbergMarquardt optimization (Levenberg, 1944, Marquardt, 1963), Trust-Region-Reflective optimization can handle bound constraints on the optimization space.

A pose normally has six degrees of freedom (DOF), three for the translation and three for the rotation. As a result of the scale ambiguity, this is reduced to five DOF for the relative pose.

Because of the normalization $\|\mathbf{t}\|=1$, all possible solutions for $\mathbf{t}$ are on the unit sphere around the first camera. Hence, $\mathbf{t}$ can be expressed in spherical coordinates $(\theta, \phi)$. Together with the rotation angles $r_{x}, r_{y}$ and $r_{z}$, the optimization space is $\left(\theta, \phi, r_{x}, r_{y}, r_{z}\right)$. As the optimization step only finds a local minimum of the reprojection error, a good initial guess is important in order to find the global minimum. Thus, the starting point for the optimization task is the relative pose as computed before.

Depending on the application, further constraints may exist, which reduce the dimension of the optimization space. For example, in a room equipped with PTZ cameras, $r_{z}$ (roll) can be fixed to 0 .

\subsection{Solving the Scale Ambiguity}

When given only two views of a scene, the solution for the scale ambiguity problem requires more information on the scene itself or the objects located in it. Our approach uses the knowledge of reference objects that have been detected, using SIFT, in both views. The best results can be obtained with planar objects, such as posters or pictures. Nevertheless, non-planar objects are also possible as reference objects, but require certain restrictions or more complex processing of the local features to achieve similar results.

The reference objects are detected by matching an image of each reference object with both views and calculating the projective transformations of the objects. The matching is done with SIFT features. This is an advantage since the SIFT features calculated for the estimation of the relative pose can be reused. The projective transformations are homographies between the the reference objects and their occurances in the two views. A homography is a pro- jective transformation that maps points on one plane to another plane (which is also the reason why planar reference objects yield the best results). It is formalized by a $3 \times 3$ matrix $\mathbf{H}$, called the homography matrix. An algorithm to compute the homography is the direct-linear-transformation algorithm (Hartley and Zisserman, 2004, p. 88), which can be combined with RANSAC to ensure robustness against outliers.

Let $\mathbf{H}=\left[\mathbf{h}_{\mathbf{1}}, \mathbf{h}_{\mathbf{2}}, \mathbf{h}_{\mathbf{3}}\right]$ be the homography matrix between the image of the reference object and one of the views and $\mathbf{K}$ the intrinsic calibration matrix of the camera. According to (Zhang, 2000) the extrinsic parameters $[\mathbf{R} \mid \mathbf{t}]$ of the camera image in relation to the reference object can be computed as

$$
\begin{aligned}
\mathbf{r}_{\mathbf{1}} & =\lambda \mathbf{K}^{-1} \mathbf{h}_{\mathbf{1}}, \\
\mathbf{r}_{\mathbf{2}} & =\lambda \mathbf{K}^{-1} \mathbf{h}_{\mathbf{2}}, \\
\mathbf{r}_{\mathbf{3}} & =\mathbf{r}_{\mathbf{1}} \times \mathbf{r}_{\mathbf{2}}, \\
\mathbf{t} & =\lambda \mathbf{K}^{-1} \mathbf{h}_{\mathbf{3}},
\end{aligned}
$$

with

$$
\lambda=\frac{1}{\left\|\mathbf{K}^{-1} \mathbf{h}_{\mathbf{1}}\right\|}=\frac{1}{\left\|\mathbf{K}^{-1} \mathbf{h}_{\mathbf{2}}\right\|}
$$

and the rotation matrix $\mathbf{R}=\left[\mathbf{r}_{1}, \mathbf{r}_{\mathbf{2}}, \mathbf{r}_{\mathbf{3}}\right]$.

The distance of the reference object (more exactly the origin of the reference object's image) to the camera center is given by $\|\mathbf{t}\|$. If the size of the object is known in the units of the world coordinate system (e.g. $\mathrm{mm}$, as used in the following), then $\mathbf{t}$ can also be expressed in this unit. Let $\mathbf{d}_{\mathbf{p} \mathbf{x}}$ be the size vector (width and height) of the reference object's image in pixels, and $\mathbf{d}_{\mathbf{m m}}$ the size vector in millimeters. Thus, the translation vector $\mathbf{t}$ scaled to millimeters is then given as

$$
\hat{\mathbf{t}}=\frac{\left\|\mathbf{d}_{\mathbf{m m}}\right\|}{\left\|\mathbf{d}_{\mathbf{p x}}\right\|} \cdot \mathbf{t} .
$$

Let $\mathbf{x}$ be the origin of the reference object's image in the scene. It can be reconstructed with the estimate of the relative pose of the second camera as computed previously. Thus, relating the translation vectors of the reference object $\hat{\mathbf{t}}_{\mathbf{1}}$ and $\hat{\mathbf{t}}_{\mathbf{2}}$ to the distances between $\mathbf{x}$ and the camera centers $\mathbf{c}_{\mathbf{1}}$ and $\mathbf{c}_{\mathbf{2}}$ provides us with two scaling factors:

$$
s_{1}=\frac{\left\|\hat{\mathbf{t}}_{\mathbf{1}}\right\|}{\left\|\mathbf{x}-\mathbf{c}_{\mathbf{1}}\right\|} \text { and } s_{2}=\frac{\left\|\hat{\mathbf{t}}_{\mathbf{2}}\right\|}{\left\|\mathbf{x}-\mathbf{c}_{\mathbf{2}}\right\|} .
$$


These scaling factors are, in theory, equal, but usually differ slightly when estimated on real data. There are two possibilities to use these factors to correctly scale the yet unscaled position of the second camera:

$$
\begin{aligned}
& \hat{\mathbf{c}}_{\mathbf{2} \mathbf{a}}=\frac{1}{2}\left(s_{1}+s_{2}\right) \cdot \mathbf{c}_{\mathbf{2}}, \text { and } \\
& \hat{\mathbf{c}}_{\mathbf{2} \mathbf{b}}=s_{1}\left(\mathbf{x}-\mathbf{c}_{\mathbf{1}}\right)+s_{2}\left(\mathbf{x}-\mathbf{c}_{\mathbf{2}}\right) .
\end{aligned}
$$

The first possibility is straightforward and applies the average scaling factor directly to the relative position of the second camera. The second possibility applies the scaling factors to the vectors between the camera centers and the reference object. This has proven to work better in scenes in which the reference object is reliably and precisely detected, giving very accurate scaling factors. In contrast, Eq. 11 achieves good results for scenes in which the relative pose is very precise, while the scaling factors are prone to noise, which may occur with small reference objects.

\section{EVALUATION}

The evaluation of our system is divided into two parts, synthetic and real benchmarks. The synthetic tests evaluate the single components of our system, the estimation of the relative pose and the calculation of the scale. To test our algorithm on real data, we used the data sets presented in (Strecha et al., 2008) and images recorded by ourselves in a smart room at our university.

\subsection{Synthetic Data}

At first, we evaluated the relative pose estimation algorithm to determine the influence of the optimization on the result. For this purpose, we generated 200 random 3D points in a box of side length 2 , centered at $\left(\begin{array}{lll}0 & 0 & 3\end{array}\right)^{\mathrm{T}}$. We then projected these points on two virtual cameras with intrinsic calibration matrices $\mathbf{K}=I$. The second camera was positioned randomly on the unit sphere. For each position, the pose was estimated 1000 times. We set the threshold of RANSAC to 1000 iterations. To test the influence of noise, we introduced white Gaussian noise of a certain signal-to-noise ratio to the projected points. More descriptively, on a $640 \times 480$ px picture, a noise level of $50 \mathrm{~dB}$ would be equivalent to a standard deviation of about $1.4 \mathrm{px}, 30 \mathrm{~dB}$ to $14 \mathrm{px}$ and $20 \mathrm{~dB}$ to $43 \mathrm{px}$. Outliers were inserted by selecting a certain percentage of points and assigning them to other points.

The performance for different percentages of outliers is unaffected by the optimization. This is due to the fact, that outliers are removed by RANSAC when

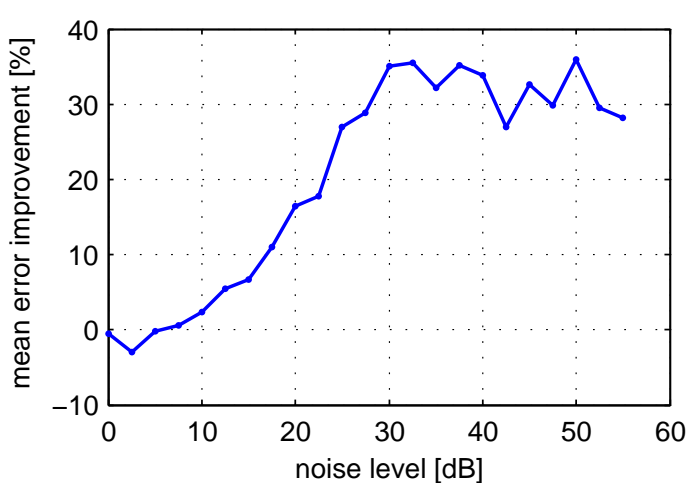

Figure 4: For noise levels starting at $25 \mathrm{~dB}$, the optimization reduces the translation error by an average of $31 \%$.

calculating fundamental matrix. The optimization afterwards does not affect the point correspondences. We obtain very robust results up to a outlier percentage of $60 \%$, with a translation error of less than $3^{\circ}$ and a rotation error of less than $1^{\circ}$.

Figure 4 shows the improvement brought by the optimization of the relative pose in the presence of noise. The translation error is reduced by $31.6 \%$ for noise levels starting at $25 \mathrm{~dB}$. The rotation error is largely unaffected by the optimization.

To test, how accurate the distance of a reference object can be estimated, a square reference object with side lengths of $50 \mathrm{~cm}$ was rotated around its yaxis and positioned on different distances along the $\mathrm{z}$-axis. For every position and rotation an image was created with a virtual camera with a focal length of 2000 and principal point $[1000,1000]^{\top}$. The reference object's distance was retrieved as described in section 3.3 including its detection using SIFT and RANSACbased homography estimation. Each calculation was repeated 100 times. The results, illustrated in Fig. 5. show that the distance can be robustly computed for all positions and rotations up to $30^{\circ}$. The error is less than $4 \mathrm{~cm}$ and for most measurements less than $2 \mathrm{~cm}$. Without rotation, the error is never above $1 \mathrm{~cm}$. For a

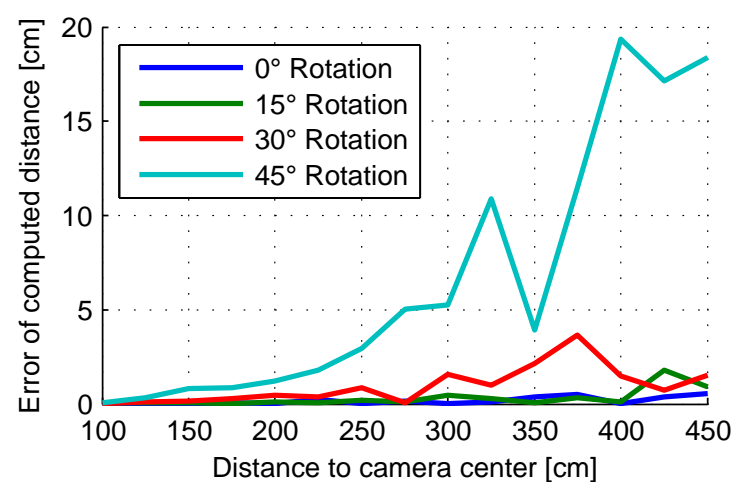

Figure 5: The graph shows the error of the calculated distance of a square reference object for different rotations. 
rotation of $45^{\circ}$, the error increases to about $20 \mathrm{~cm}$ at a distance of $4 \mathrm{~m}$ to $4.5 \mathrm{~m}$. This is still less than $5 \%$ of the distance of the reference object.

\subsection{Real Data}

Furthermore, we evaluated our proposed approach on different real data sets. The first tests use the Herz-Jesu-P8 and fountain-P11 data sets (see (Strecha et al., 2008)). We chose these data sets, because they contain images with a high resolution and are well annotated. We generated reference objects for both data sets by cropping parts of the images, that showed walls which were visible in most pictures. We reduced the data sets to those images, in which the reference object could be detected. The camera poses were calculated iteratively. Figure 6 shows the reconstruction results and position errors for both data sets.

Since the camera poses are iteratively estimated, small errors are propagated and increase in the process of the computation. Still, the results are very accurate and the errors are in the range of centimeters. Over a total distance of $15.5 \mathrm{~m}$ in data set HerzJesu-P8, the error of the last camera is only $12 \mathrm{~cm}$, or $0.77 \%$. The distance between the first and last camera in fountain-P11 is $11 \mathrm{~m}$, yet the position error is a mere $6 \mathrm{~cm}$, or $0.55 \%$. The data sets have certain characteristics that help attaining such a high precision: high resolution, small baselines between the cameras and highly textured scenes that produce well distributed point correspondences.

These characteristics are less present in the data recorded in our smart environment, a room of roughly $25 \mathrm{~m}^{2}$. It has several microphone arrays, computer controlled lighting and, most importantly for our case, four ceiling-mounted PTZ cameras with a resolution of $752 \times 596$ pixels. The position and rotation of the PTZ cameras are known. Pictures were taken with a similar PTZ camera on a tripod at 23 different positions in the room. The positions were measured by hand in the environment's coordinate frame to obtain a ground truth. For each position, the absolute camera pose was calculated several times using one of the known cameras. The results with the lowest reprojection error for every image were selected and aggregated by using the mean and the median of the coordinates and then compared to the ground truth. Three posters, two of size $118.9 \times 84.1 \mathrm{~cm}$ and one of size $60 \times 91 \mathrm{~cm}$, were chosen as reference objects. Table 1 shows the attained results.

We arrive at a mean position error of $41.30 \mathrm{~cm}$ for the mean position and $39.50 \mathrm{~cm}$ for the median position. Compared to the much lower median position error of $25.24 \mathrm{~cm}$, and $21.64 \mathrm{~cm}$ respectively, we see that the camera was very poorly localized for several mea- surement points, with an error of over $2 \mathrm{~m}$ for camera position 3 and about $1 \mathrm{~m}$ for positions 16 and 17 . The poses for other positions were very close to the ground truth, often with an error of less than $16 \mathrm{~cm}$.

There are different reasons for the high errors in the detected poses for the images in our smart room. Most importantly, the cameras provide noisy images without much detail and fail to record fine structures. Furthermore, the environment itself does not contain much details, making it hard to find reliable point correspondences. This is made even more difficult by the the low number of cameras and big viewpoint changes between the cameras. As a consequence, point correspondences were often found mostly between the projections of the reference objects and for this reason only locally distributed in the images. Although the error seems relatively large on the first sight especially when compared to the results achieved on Herz-Jesu-P8 and fountain-P11 -, we consider a median error of $21.64 \mathrm{~cm}$ an acceptable result due to the difficulty of the environment. Furthermore, we have to consider the possible influence of minor ground truth measurement errors that were, for example, introduced by the unknown exact location of the focal point within the PTZ camera casing.

\section{CONCLUSION}

We proposed a new approach to compute the global scale between two views given a known reference object. To this end, we first calculate the relative pose with established methods of epipolar geometry. We then reduce the reprojection error of the pose with a nonlinear optimization step, significantly improving the result. Afterwards, the overall scale of the scene is reliably estimated by detecting a known reference object in both camera views. Through different tests, our system has proven to very precisely compute absolute poses. In two data sets with small distances between camera images, we achieved an absolute error for the iterative pose estimation of less than $1 \%$. The global localization of cameras in our conference room was less accurate, hindered by low resolution cameras, plain walls without much detail and wide baselines. However, we still achieved an accuracy of about $20 \mathrm{~cm}$ for most camera pairs, making our system a useful and convenient technique for the extrinisc calibration of multiple cameras.

\section{ACKNOWLEDGEMENTS}

This work is partly supported by the German Research Foundation (DFG) within the Collaborative 


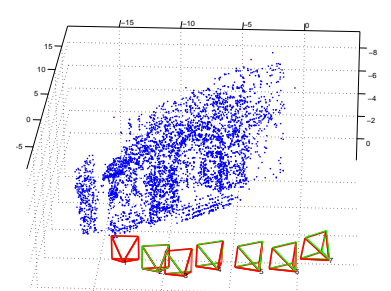

(a)

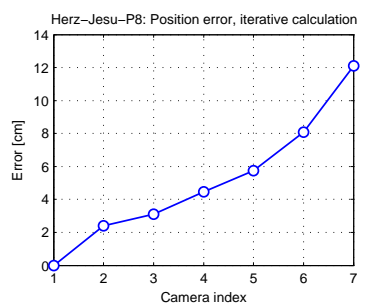

(b)

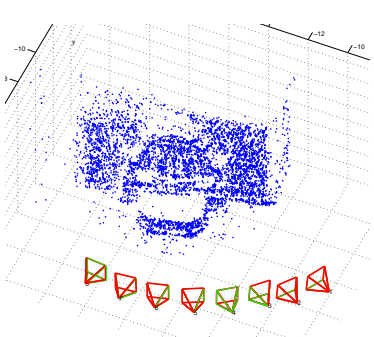

(c)

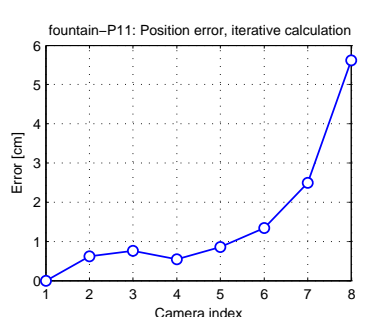

(d)

Figure 6: The scene reconstruction and position error of data sets Herz-Jesu-P8 (a-b) and fountain-P11 (c-d). Red cameras show the iteratively calculated camera poses, green cameras the ground truth (might not be distinguishable from the calculated poses in this scale). The scene reconstruction was achieved without further optimization, such as Bundle Adjustment.

\begin{tabular}{|c|c|c|c|c|c|c|c|c|c|}
\hline & \multicolumn{4}{|c|}{ Error of mean position $[\mathrm{cm}]$} & \multicolumn{4}{|c|}{ Error of median position $[\mathrm{cm}]$} & \multirow{2}{*}{$\begin{array}{l}\text { mean reprojection } \\
\text { error }[\mathrm{px}]\end{array}$} \\
\hline & total & $\mathrm{x}$ & $\mathrm{y}$ & $\mathrm{z}$ & total & $\mathrm{x}$ & $\mathrm{y}$ & $\mathrm{z}$ & \\
\hline mean & 41,30 & 19,97 & 18,78 & 25,54 & 39,50 & 17,11 & 18,32 & 24,26 & 0,7914 \\
\hline median & 25,24 & 8,27 & 8,65 & 11,29 & 21,63 & 6,64 & 7,77 & 12,11 & 0,6972 \\
\hline standard deviation & 48,96 & 34,72 & 25,61 & 29,19 & 49,74 & 35,18 & 26,51 & 29,88 & 0,3897 \\
\hline $\min$ & 8,06 & 0,20 & 0,02 & 0,10 & 5,31 & 0,97 & 0,10 & 0,10 & 0,3534 \\
\hline $\max$ & 227,76 & 165,60 & 117,74 & 102,89 & 227,76 & 165,60 & 117,74 & 102,89 & 1,8259 \\
\hline
\end{tabular}

Table 1: Errors for the estimated poses of 23 images taken in our smart room.

Research Program SFB 588 "Humanoide Roboter". The authors would like to thank Manel Martinez and Jan Richarz for their helpful comments.

\section{REFERENCES}

Aslan, C. T., Bernardin, K., and Stiefelhagen, R. (2008). Automatic calibration of camera networks based on local motion features. ECCV-M2SFA2.

Brückner, M. and Denzler, J. (2010). Active self-calibration of multi-camera systems. Proc. DAGM.

Coleman, T. F. and Li, Y. (1996). An Interior Trust Region Approach for Nonlinear Minimization Subject to Bounds. SIOPT, 6(2):418-445.

Frank-Bolton, P. et al. (2008). Vision-based localization for mobile robots using a set of known views. Proc. ISVC, pages 195-204.

Hartley, R. I. and Zisserman, A. (2004). Multiple View Geometry in Computer Vision. Cambridge University Press, second edition.

Levenberg, K. (1944). A Method for the Solution of Certain Problems in Least-Squares. Quart. Applied Math., 2:164-168.

Liu, J. and Hubbold, R. (2006). Automatic camera calibration and scene reconstruction with scale-invariant features. Proc. ISVC, pages 558-568.

Lowe, D. G. (1999). Object recognition from local scaleinvariant features. Proc. IEEE ICCV, pages 11501157.

Lowe, D. G. (2004). Distinctive image features from scaleinvariant keypoints. IJCV, 60(2):91-110.

Marquardt, D. W. (1963). An Algorithm for LeastSquares Estimation of Nonlinear Parameters. SIAP, 11(2):431-441.
Nistér, D. (2004). An efficient solution to the five-point relative pose problem. TPAMI, 26:756-777.

Rodehorst, V., Heinrichs, M., and Hellwich, O. (2008). Evaluation of relative pose estimation methods for multi-camera setups. Proc. Congress ISPRS.

Salah, A., Morros, R., Luque, J., Segura, C., Hernando, J., Ambekar, O., Schouten, B., and Pauwels, E. (2008). Multimodal identification and localization of users in a smart environment. JMUI, 2(2):75-91.

Schauerte, B., Richarz, J., Plötz, T., Thurau, C., and Fink, G. A. (2009). Multi-modal and multi-camera attention in smart environments. Proc. ICMI.

Snavely, N., Seitz, S. M., and Szeliski, R. (2008). Modeling the world from internet photo collections. IJCV, 80(2):189-210.

Stewénius, H., Engels, C., and Nistér, D. (2006). Recent developments on direct relative orientation. ISPRS, 60:284-294.

Strecha, C., von Hansen, W., Gool, L. V., Fua, P., and Thoennessen, U. (2008). On benchmarking camera calibration and multi-view stereo for high resolution imagery. CVPR, 0:1-8.

Svoboda, T., Martinec, D., and Pajdla, T. (2005). A convenient multi-camera self-calibration for virtual environments. PRESENCE: Teleoperators and Virtual Environments, 14(4):407-422.

Voit, M. and Stiefelhagen, R. (2010). 3-D user-perspective, voxel-based estimation of visual focus of attention in dynamic meeting scenarios. ICMI-MLMI.

Xiong, Y. and Quek, F. (2005). Meeting room configuration and multiple camera calibration in meeting analysis. Proc. ICMI.

Zhang, Z. (2000). A flexible new technique for camera calibration. TPAMI, 22(11):1330-1334. 